\title{
Chemistry of Photosynthesis
}

$\mathrm{I}^{\mathrm{N}}$ his presidential address to the tenth annual general meeting of the Indian Chemical Society held at Bombay, Prof. N. R. Dhar discussed some aspects of the chemistry of photosynthesis ("Chemical Aspects of Carbon Assimilation", J. Indian Chem. Soc., 11,145 ; 1934). Numerous experiments carried on in his laboratory during the past ten years show that formaldehyde is synthesised and detected when dilute solutions ( 5 per cent) of bicarbonates of the alkali metals are exposed to sunlight for about four hours in thin layers $(0.5 \mathrm{~cm}$. thick) either in open dishes or covered with silica plates at temperatures up to $30^{\circ}$; higher temperatures are prejudicial to formaldehyde formation. The amount of formaldehyde photosynthesised per 100 c.c. of solution exposed is $0 \cdot 00007-0.0001 \mathrm{gm}$. Schryver's reagent is most sensitive for the detection of formaldehyde in small quantities. The amount of formaldehyde obtained by exposing the bicarbonate solutions in the same dishes placed in a bath at $40^{\circ}$ is about one third of that obtained at $30^{\circ}$ under identical conditions.

It is interesting to note that in Nature the amount of carbon assimilation is less at $40^{\circ}$ than at $30^{\circ}$. Moreover, the amount of formaldehyde in rain-water obtained after a succession of bright days is practically the same as is photosynthesised by exposing potassium bicarbonate solutions to sunlight. Larger amounts of formaldehyde are produced by the reduction of bicarbonate or carbonic acid solutions by metallic cerium, tungsten, iron, etc. This reduction is accelerated by light. These results have been confirmed by Mezzadroli and his collaborators (1927-29) and very recently by Yoe and Wingard ( $J$. Chem. Phys., $1,886 ; 1933)$.

Reducing sugars have been obtained by exposing formaldehyde solutions to sunlight mixed with ferric chloride. The temperature coefficient for this reaction for a $10^{\circ}$ rise of temperature is of the same order as that of the photosynthesis with Elodea or Hydrilla.

When solutions of glycol and potassium nitrate are exposed to sunlight for 8 hours in presence of titanium oxide as a photocatalyst, glycine is produced. Similarly, a solution containing glucose and potassium nitrate with titanium oxide, when exposed to sunlight for the same period, appears to produce arginine amongst other substances. Longer exposure to light causes the disappearance of the amino acids photosynthesised, probably due to their photooxidation. Solutions of ammonium lactate also form amino acids on exposure to light. The amino acids thus obtained can be readily detected by the valuable 'ninhydrin' test.

The temperature coefficients of photosynthesis in plants do not obey the Arrhenius relation connecting the temperature and the velocity of a reaction. In photosynthesis, the observed values of temperature coefficients are always smaller than the calculated ones. The phenomenon of 'solarisation', or the adverse effect of strong light on photosynthesis, and the fact that the compensation point, that is, the light intensity at which the photosynthetic and respiratory activities of the plant compensate each other, decreases with fall of temperature. The nonapplicability of the Arrhenius relation to photosynthesis has been explained on the following considerations :-

(a) Photosynthesis is proportional to the light intensity.

(b) Respiration takes place in the dark but is appreciably accelerated by light.

(c) An increase of temperature enhances respiration more markedly than photosynthesis.

Moreover, the smaller temperature coefficient of photosynthesis in feeble than in intense light and the lower temperature coefficient in chlorophyll-poor leaves in comparison with chlorophyll-rich leaves have also been explained from the same viewpoint.

A new theory of carbon assimilation is also advanced in which the following are the main points : (1) Partial activation of carbon dioxide and water at the leaf surface due to their adsorption by chlorophyll and other pigments. It seems that chlorophyll and carotinoids act as photo-sensitisers, and as reducing agents in the photo-reduction of carbon dioxide.

(2) Further activation of the adsorbed carbon dioxide and water by absorption of a part of the energy available from respiration and oxidation of carotin, and the formation of activated carbon dioxide and water as products of respiration.

(3) Absorption of light by chlorophyll and other pigments and the dissociation of the activated water molecules, on the leaf surface, into $\mathrm{H}$ and $\mathrm{OH}_{1}$, and the reduction of activated carbon dioxide into formaldehyde by the atomic hydrogen produced from the sensitised photolysis of water. The amount of energy required to decompose a gm. mol. of water into $\mathrm{H}$ and $\mathrm{OH}$ is practically the same as that necessary for the formation of a $\mathrm{gm}$. mol. of formaldehyde from carbon dioxide and water.

(4) Polymerisation of formaldehyde to reducing sugars.

(5) The formation of hydrogen peroxide from $\mathrm{OH}$ and the rapid decomposition of hydrogen peroxide into water and oxygen on the leaf surface. Formaldehyde is obtained more readily by the photo-oxidation of energy-rich compounds than by exposing bicarbonate solutions to light.

There is an intimate relation between respiration and photosynthesis in the plant kingdom. It seems that photosynthesis cannot proceed without the energy available from respiration for the partial activation of carbon dioxide and water. The need of oxygen in photosynthesis is also easily explained. Experimental evidence in support of this theory of carbon assimilation was given.

\section{Botanical Work in the University of Lucknow}

$\mathrm{T}$ HE University of Lucknow Department of Botany issues a small pamphlet which contains abstracts of the published work of the Department in the quinquennium 1928-32. This report makes it clear that the contributions of this Department, under the guidance of Prof. Birbal Sahni, have been very valuable in nature and cover such a wide field of botanical activity that the senior students of the Department should readily find both inspiration and guidance whatever special interest may determine the approach to their botanical problems.

Prof. Sahni himself is represented by a long series 